\title{
Réception et dilution des apports de la pensée des Freinet. Le cas des militants pédagogiques parisiens après 1945
}

Xavier Riondet, Université de Lorraine

L'Éducation nouvelle se compose de différents courants et écoles de pensée. Comment ces collectifs ont-ils évolué et perduré au fil des années? Nous prenons ici l'exemple du Mouvement Freinet français. En interrogeant le processus de réception de la pensée originale des Freinet datant des années 1930 par les militants parisiens des années 1960, nous proposons une lecture argumentée des controverses et divergences internes au Mouvement Freinet à l'aube de l'émergence d'une nouvelle aventure de la pédagogie institutionnelle.

L'institutionnalisation de l'Éducation nouvelle s'est caractérisée par l'émergence de différents courants et mouvements spécifiques, dont certains ont voulu faire école autour d'un qualificatif, d'un nom et d'une référence à une expérience qui s'était déroulée dans le contexte de l'Entre-deux-guerres. La pédagogie de l'École Freinet à Vence a indéniablement constitué un des multiples styles pédagogiques ayant marqué l'histoire de l'Éducation nouvelle pendant les années 1930. Cet article s'intéresse à la réception de cette expérience au sein du Mouvement Freinet français dans un contexte où les références à l'Éducation nouvelle commencent à subir la concurrence des sciences humaines et sociales (Savoye, 2004). Il s'agit d'un moment emblématique pendant les années 1960, où, sous fond de conviction partagée, des controverses sont apparues, donnant lieu à de nouvelles conceptions, des scissions et parfois des ruptures au sein du Mouvement Freinet. Cet épisode particulier (l'émergence de la pédagogie institutionnelle au sein de ce mouvement) a déjà été abordé par le passé (Bruliard \& Schlemminger, 2012; Dubois, 2019; Fonvielle, 1989; Hess, 1975; Hess \& Savoye, 1993; Michaud, 1969; Pain, 1979) mais la consultation récente d'archives méconnues liées à l'histoire de l'École Freinet autorise à revenir aujourd'hui sur cet épisode en prenant davantage en compte le point de vue de ceux qui n'ont pas survécu, physiquement ou symboliquement, à la montée en puissance de la pédagogie institutionnelle dans les années 1960-1970. L'enjeu de l'analyse est d'observer comment une des expériences pédagogiques majeures de l'Éducation nouvelle de 
l'Entre-deux-guerres a pu être reçue, plusieurs décennies plus tard, par de jeunes enseignants désireux de réformer l'école. Entre l'École Freinet ouverte à Vence en 1936 et le Mouvement Freinet actuel se situent une histoire complexe tiraillée entre deux stratégies: vivre caché et rester heureux, ou croître, se développer au prix de simplifications et de quiproquos. Passer d'un réseau relativement fermé et homogène à un mouvement de masse est donc une question bien connue des militants, mais qui n'est pas sans controverses. Cela demande au chercheur de s'attarder sur l'espace conflictuel que représente en lui-même le mouvement, pour s'intéresser dans les périodes de passation, de transition et d'héritages, à la question des différends, des conflits et des ruptures (Go, 2015; Riondet, 2015; Go \& Riondet, 2019).

La détérioration des rapports entre Célestin Freinet (1896-1966) et certains militants parisiens débouche sur deux crises célèbres (Go \& Riondet, 2019; Riondet, 2015). En 1961, le départ de Raymond Fonvieille (1923-2000) et Fernand Oury (1920-1998) donne lieu à une autre aventure collective: les débuts de la pédagogie institutionnelle. Quelques années plus tard, une brochure intitulée Contribution à l'histoire du Mouvement Freinet signée par Roger Bonbonnelle, Michel Faligand et Jacques Gilbert (1966) met le feu aux poudres dans le mouvement, mettant à mal Freinet et débouchant sur de nouvelles défections. Ces épisodes de l'histoire de la "Pédagogie Freinet» posent la question propre à de nombreux mouvements pédagogiques: que devient la référence originaire à la base d'un collectif lorsque ce dernier traverse les âges? Notre texte aspire à objectiver les causes profondes d'une rencontre manquée, et ce qui obstrue sans doute inévitablement la transmission interne à tout mouvement pédagogique. Notre étude se propose d'amorcer une analyse des conditions objectives des relations entre Freinet et ces contradicteurs des années 1960 en partant de l'idée que le Mouvement Freinet peut être abordé comme un "champ», c'est-à-dire un espace de positions fonctionnant avec des règles spécifiques, dans une relative autonomie, et dans lequel des forces déterminent des conduites et des luttes ont lieu pour conserver ou transformer ce champ de forces (Bourdieu, 2015, 2016). Dans ce cadre, on peut chercher à analyser les relations et les controverses à partir de concepts connexes comme les «trajectoires» et les «capitaux».

\section{5, une nouvelle conjoncture et de nouvelles stratégies}

Tout champ a ses limites et ses frontières. Lors des premières années de regroupement autour de Freinet, la délimitation entre celles et ceux faisant partie de ce champ et les personnes qui lui sont extérieures est parfaitement claire. Au cours des années 1930, Freinet se revendique «éducateur prolétarien» (Go \& Riondet, 2019). Cela désigne une position parfaitement délimitée dans une conjoncture. Suite à la mise à l'écart de Freinet de l'Éducation Nationale et à la tuberculose 
d'Élise Freinet (1898-1983), tous deux créent une école spécifiquement dédiée aux enfants du prolétariat pour faire face à l'école de classe que constitue l'École publique (Riondet, 2016). Autour d'eux, on retrouve un cercle de proches partageant croyances, visions du monde, aspirations sociales et convictions politiques. L'école nouvelle et privée de Vence tire profit des apports de l'Éducation nouvelle et du naturisme révolutionnaire (Riondet, 2016), et Freinet cherche alors à rallier au local, paysans et ouvriers, dans un front de lutte contre les forces capitalistes et réactionnaires, en diffusant un cadre théorique rendant compte de l'exploitation et permettant de visualiser les résistances et luttes possibles (Riondet, 2019). L'éducation des enfants (et leur éventuel passage par l'École Freinet à Vence) représente un des aspects de la lutte "classe contre classe»; il s'agit aussi de réfléchir à d'autres manières de consommer, de produire, de se loger, et de vivre (Riondet, 2016).

\section{Création de L'ICÉM}

Le mode de pensée et les intimes convictions des Freinet n'ont pas disparu après 1945 mais l'heure est à la reconstruction sociale, économique et scolaire. Pour Freinet, il s'agit de faire proliférer l'expérience de Vence (Go \& Riondet, 2019). Celle-ci est au cœur des propositions de réforme qu'il diffuse au lendemain de la Seconde guerre mondiale. Les Freinet sont parfaitement conscients qu'il leur faut trouver une solution pour pérenniser cette école (Go \& Riondet, 2019). Par ailleurs, il s'agit toujours de faire évoluer la société d'un point de vue social. Freinet s'estime rapidement lésé dans la mise en réseau et les débats orchestrés dans le cadre de la commission Langevin-Wallon qui s'attèle de 1944 à 1947 à l'élaboration d'une réforme scolaire d'envergure (Go \& Riondet, 2019).

L'émergence de l'Institut Coopératif de l'École Moderne (ICÉM) en 1947 a pour enjeu d'institutionnaliser les forces pédagogiques autour de Freinet. Celui-ci décide de créer un «Institut» pour rassembler "la masse des instituteurs dynamiques» (Freinet, 1946b, p. 169), institut dont le nom ne fut pas arrêté tout de suite puisque l'idée de ce regroupement apparut en 1946 sous la dénomination «Institut Central de l'École Moderne». Le problème est de savoir si cette organisation doit être une fédération de groupes départementaux ou un pilotage de ces groupes à partir d'un bureau central situé à Cannes et Vence. Ce questionnement est récurrent dans l'histoire du Mouvement Freinet. Dès le départ, l'ICÉM est traversé de cette tension concernant la légitimité de son pilotage. Finalement, Freinet avait décidé d'opter pour la notion d'institut coopératif puisqu'il fit porter la mention, sur la couverture de L'Éducateur, de «Revue pédagogique de l'Institut Coopératif de l'École Moderne».

Une partie des militants ne sont guère favorables au caractère centralisé d'une organisation sous la direction de Freinet. Ce dernier recherche finalement une position d'équilibre afin de maintenir sa direction sur le mouvement: "Que nos adhérents s'organisent départementalement; qu'ils s'inscrivent nationalement à l'institut Coopératif de l'École Moderne» (Freinet, 1946c, 2e de couverture). 
Freinet indique que cet institut organiserait le mouvement au plan international de «l'École Moderne», ce qui le démarque définitivement du mouvement de l'Éducation nouvelle pour développer son propre courant. Toutefois, il garde l'initiative de son œuvre propre en précisant que «cette expérience est spécifiquement française, puisqu'elle est fille du milieu français» (Freinet, 1946a), cette expérience mêlant la matérialisation dans l'École Freinet d'une forme de pensée, la mise en réseau de militants, l'organisation de stages de formation, la mise en place d'espaces de publication et de diffusion de matériel pédagogique. La volonté d'organisation en jeu dans la création de l'ICÉM comporte deux versants: une stratégie défensive (maintenir une situation, préserver des acquis, protéger l'École de Vence) et une stratégie offensive pour diffuser, gagner du terrain dans le monde de l'éducation (Freinet, 1946b).

\section{Sortie du film L'École Buissonnière}

La sortie d'un film de propagande va contribuer à essaimer les idées de Freinet tout en essayant de fidéliser de nouveaux publics. Le film L'École Buissonnière, réalisé par Jean-Paul Le Chanois (1909-1985), se distingue par un récit édulcoré de l'affaire Saint-Paul de Vence qui est l'occasion de fédérer les forces progressistes pédagogiques et le grand public derrière un instituteur mobilisé en faveur de l'enfance qui est censé rappeler l'histoire de Freinet. Les historiens de la pédagogie ont finalement peu évoqué le stratège Freinet, mais à travers ces deux événements (création de l'ICÉM et sortie d'un film de propagande), ce dernier organise autour de lui un mouvement de masse pour diffuser ses techniques, accroître son influence, tout en plaçant l'École Freinet au cœur du mouvement pédagogique, et en lui cherchant un statut dans le paysage institutionnel scolaire.

Le challenge animant Freinet est le suivant: comment accroître l'influence du mouvement en gardant la main sur son identité? Question légitime car ce mouvement est originellement lié à une expérience dans laquelle les Freinet étaient parties prenantes et qu'ils connaissaient mieux que quiconque. Le challenge évoqué n'est pour autant pas gagné d'avance puisque le développement de l'ICÉM rencontre trois éléments: évolution de la société (tant culturelle que technologique), évolution socio-politique et évolution de la problématisation socio-éducative. La transmission de l'identité «Freinet» à destination des nouveaux membres du mouvement ne pouvait être qu'impactée par ces différentes évolutions.

\section{Freinet, Vence et L'IPÉM: une rencontre impossible?}

En France, les réformes scolaires entre 1945 et 1975 ont pour effets de prolonger la scolarisation des élèves et de regrouper différents cursus de scolarisation dans un même établissement. Le Mouvement Freinet, et son fondateur en premier 
lieu, cherche à tirer profit de ces évolutions tout en essayant de défendre sa vision de la pédagogie et de l'École. Cependant, Freinet s'appuie sur des contextes régionaux très disparates avec des réseaux locaux parfois très différents dans un contexte sociétal en pleine évolution. Pour saisir la réception de la pensée des Freinet au sein de certains territoires du Mouvement Freinet, il faut prendre en compte l'évolution globale de la société française dans laquelle se déroulent les échanges et débats en jeu.

\section{La France des Trente Glorieuses}

Beaucoup de jeunes militants des mouvements pédagogiques sont aux prises de réalités sociologiques nouvelles dans un paysage français en pleine évolution. Le paysage français vit de profondes mutations. Les «Trente Glorieuses» (19461975) marque l'avènement de la société de consommation. Alors que de 1946 à 1975 le revenu national disponible par habitant a presque doublé (Borne, 1988, p. 41) se diffusent progressivement les quatre "produits symboles» de cette période: le réfrigérateur, l'automobile, la télévision et le lave-linge.

On assiste également à une croissance des villes, et en particulier de l'agglomération parisienne. Rétrospectivement, l'historien Georges Dupeux (1920-1994) écrit d'ailleurs: «La concentration de la population dans la région parisienne est un des faits majeurs de l'histoire de la population française» (1972, p. 23). La hausse de la natalité conjuguée à une baisse de la mortalité (notamment infantile) donne lieu à un accroissement de la population française (de 40,1 millions en 1946 à 45 millions à la fin des années 1950). Entre 1954 et 1975, la population des villes de 5000 habitants s'accroît de 13 millions de personnes, mais la composition de la population évolue également: un million de rapatriés d'Afrique du Nord arrivent en France entre 1950 et 1962 et l'immigration augmente substantiellement entre 1954 et 1975 , passant de 1,7 million à 3,5 millions d'individus.

Ces différents phénomènes ont des répercussions particulièrement problématiques au niveau du mode de vie, notamment en Île-de-France. En 1946, dans le département de la Seine, 780000 individus, ce qui représente la moitié de la population du département, vivent à trois ou plus par pièce (Borne, 1988, p. 27). C'est le début de ce qu'on va appeler l'ère des «grands ensembles»: quelques groupes d'immeubles accueillent progressivement des centaines, voire des milliers de logements (Borne, 1988, p. 48). Par ailleurs, une autre tendance globale se confirme: les paysans veulent de moins en moins vivre comme autrefois. Si l'on recense cinq millions d'agriculteurs en 1921, ils ne sont plus que deux millions en 1968. Cela se traduit parfois par le refus de prendre la suite des activités agricoles familiales, ou par le choix de ne pas y rester cantonné durant sa vie professionnelle (Asselain, 1984).

\section{La situation des "parisiens" du Mouvement}

Alors que les regroupements autour de Freinet durant l'Entre-deux-guerres se distinguaient par le refus d'une forme de civilisation et l'organisation locale 
d'autres expériences concrètes (Riondet, 2016), le Mouvement Freinet va évoluer par homologie avec ces nouvelles tendances sociales et culturelles. Fernand Oury s'auto-présente dans ses propres écrits comme officiant depuis 1939 dans des «écoles primaires de banlieue» (Oury \& Pain, 1972, p. 17), mais la délimitation de ce contexte est à l'œuvre à l'intérieur du Mouvement Freinet depuis les années 1950, comme en témoigne la revue Éducateur Île-de-France. Cet espace de publication rend compte spécifiquement des enjeux propres à l'Île-de-France. En évoquant «la situation des Parisiens de l'École Moderne» (1958/1959, p. 2), le rédacteur en chef, Raymond Fonvieille désigne le préalable de toute réflexion pédagogique en Île-de-France. Nombre d'écrits vont alors dans ce sens. On y évoque des "groupes urbains» dans lequel on observe l' «entassement» des enfants» et les «fourmilières d'enfants» (Oury, 1962, p. 2). Dans cette école urbaine en périphérie parisienne, c'est souvent l'inadaptation des élèves qui pose problème du point de vue de l'institution, et non l'inadaptation de l'école elle-même, et la situation ne cesse de se détériorer, comme le rappellent Oury et Pain: «L'école urbaine souligne et aggrave les déficiences que, selon certains, elle est censée compenser» (1972, p. 23).

Cet ancrage géographique et social est particulière visible lorsque l'on aborde la question des trajectoires des militants pédagogiques, et cela nous renseigne, par la même occasion, sur les publics scolaires en jeu. Michel Faligand, par exemple, avait préalablement exercé dans une classe annexée au service de neuropsychiatrie de la Salpêtrière avec des enfants aux profils variés ${ }^{1}$ avec des niveaux mentaux et scolaires très hétérogènes (1965). C'est dans ce contexte qu'il avait commencé par initier des réunions quotidiennes présidées par des enfants puis des techniques pédagogiques centrées sur l'expression, en se concentrant sur la dimension «motivationnelle» des apprentissages et le développement de la socialisation. Lorsque Faligand écrit dans la revue parisienne, il met justement en évidence la spécificité du public avec lequel il travaille:

Les enfants (au nombre de 15) ont des QI allant de 51 à 81, parmi eux: un épileptique, un post-encéphalitique, 2 ou 3 caractériels. Nombreux cas de dysphones. Retards scolaires plus importants que les QI ne le laissent supposer (1963, p. 17).

Si les Freinet avaient fait l'expérience de l'impossibilité de changer l'instruction publique de l'intérieur, la plupart des militants parisiens se confrontent dans les années 1960 à l'état particulièrement inquiétant de l'enseignement spécial.

\section{Perte de L'influence des Freinet}

L'évolution du Mouvement Freinet, notamment dans les réseaux parisiens, se caractérise également par une perte du capital de référence: le capital «Freinet». En effet, la manière dont s'est constitué un Mouvement Freinet de masse après 1945 s'est effectuée à partir de la circulation de représentations relativement simplistes de la pensée des Freinet. Par ailleurs, une partie des militants sont relativement jeunes et bien éloignés des références qui ont marqué la pensée des Freinet. Dans 
les colonnes de L'Éducateur Ile-de-France, Fonvieille fait le constat suivant:

De plus en plus, nous nous adressons à des jeunes et dans la région parisienne plus qu'ailleurs peut-être, à des jeunes qui n'ont jamais entendu parler de Freinet, puisque nombreux sont ceux qui n'ont pas fréquenté l'École Normale (1961a, p. 8).

Parmi les parisiens avec qui Freinet sera en désaccord, Oury est parmi les plus anciens, il a pourtant vingt-cinq années d'écart avec Freinet. Fonvieille, bien que né près d'Arras, n'a connu que l'Île-de-France: Colombes, Saint-Denis puis Gennevilliers, découvrant la "Pédagogie Freinet» après la Seconde guerre mondiale. Jacques Gilbert, enseignant le français en milieu bilingue, découvrant l'expression libre en Algérie en 1960 par le biais des publications «Freinet», se rapproche de Fonvieille en 1961, puis de l'IPÉM, lorsqu'il revient en région parisienne en prenant un poste à Gennevilliers. André Bonbonnelle (1932-2016), titulaire d'un CAP d'instituteur et d'un CAEA ${ }^{2}$, fait lui-aussi partie des jeunes du Mouvement dans les années 1960. Instituteur en Seine-et-Oise de 1956 à 1964, il découvre le Mouvement de l'École Moderne en 1963 à Niort et commence à travailler avec le Groupe Parisien. C'est suite à l'indignation à l'égard de leur contexte professionnel que rentrent dans le mouvement pédagogique ces jeunes instituteurs, et les références à Freinet et à Vence ne sont guère mises en avant lorsqu'ils se socialisent dans le collectif. Lors de la troisième réunion psycho-pédagogique organisée le 25 septembre 1958 par l'IPÉM intitulée «Action psychologique de la Coopérative dans la Classe Moderne», le postulat de départ est le suivant:

Le milieu urbain dépersonnalisé ne permet guère aux enfants d'agir librement, de faire des expériences sociales, de prendre des responsabilités.

L'école devrait jouer un rôle compensateur et fournir un milieu riche de contacts humains, de relations sociales, de possibilités d'initiative et d'expériences réelles.

Or les méthodes actives, officiellement recommandées demeurent le privilège des petites écoles rurales ou de quelques institutions privées.

Il ne s'agit pas d'un hasard (Action psychologique de la Coopérative dans la Classe Moderne, 1958, p. 1).

À cette occasion, les militants parisiens autour d'Oury se revendiquent précisément comme parisiens, instituteurs et modernes, sans références explicites à Freinet. De fait, certains ne connaissent pas l'expérience pédagogique et politique de Vence. Jeunes enseignants et militants, ils méconnaissent totalement l'histoire de Vence. Lorsque Fonvieille, censé être parmi les plus avertis du groupe parisien, se lance pour sa revue dans un petit récit historique de l'expérience de Vence, il propose un résumé très synthétique de l'ouvrage d'Élise Naissance d'une pédagogie populaire dans lequel la formulation "pédagogie prolétarienne» n'est utilisée qu'une fois: 
En 1934-1935, c'est l'installation à Vence où des paysans viennent l'aider à construire son école. Elle est debout à la fin de 1935 et Freinet y reçoit des fils d'ouvriers de la région parisienne» [...] À la libération, c'est le retour à la C.E.L. Il faut repartir à zéro (Fonvieille, 1961c, pp. 10-11).

Fonvieille considère que Vence appartient au passé et ne dit rien du contexte social et politique (école prolétarienne accueillant des enfants malades issus du prolétariat), ni des techniques éducatives (texte libre, imprimerie, conférence), des pratiques (coopération, vie communautaire, naturisme, écologie) et des implicites à l'œuvre (cure morale, régénération des enfants). Cette méconnaissance conduit la rédaction de la revue parisienne à évoquer le fait «de travailler dans les paisibles classes de campagnes ou à l'École Freinet de Vence» 3 en oubliant la situation des premiers élèves scolarisés à Vence (orphelins, malades, galeux, réfugiés, allophones, caractériels, etc.) dans des conditions pour le moins modestes et peu paisibles.

\section{Nouvelles situations, nouvelles influences}

À cette époque, plusieurs militants importent différentes références à la mode dans le monde des sciences pédagogiques. Le groupe parisien suit un effort de structuration spécifique. Progressivement se mettent en place une rubrique dans la revue Éducateur Île de France et un groupe de travail sous l'intitulé "Classes de ville». La première réunion du groupe «école de villes et techniques Freinet» a lieu le 19 octobre 1961 à Choisy (Faligand, 1961, p. 25). La conjoncture parisienne est vécue comme une situation susceptible de concerner tous les éducateurs. Il y a là une part de réalité car il y a une évolution notable de la société française, mais il y a également une part importante de franciliano-centrisme ${ }^{4}$. Les éducateurs nommés précédemment, qu'il s'agisse de Fonvieille, Oury ou Bonbonnelle, sont même persuadés de constituer une élite lucide:

Le fait qu'instituteurs laïcs de Seine et de Seine et Oise, nous exerçons dans un milieu où les problèmes concentrationnaires et administratifs nous assiègent quotidiennement et étroitement. Ce qui en fait, nous place en avant-garde (Faligand, 1961, p. 25).

Ces forces pédagogiques se connectent au Mouvement Freinet par le biais de plusieurs thèmes de travail: l'adaptation des techniques Freinet dans les écoles de villes, les problèmes individuels posés à l'instituteur utilisant les techniques Freinet dans ses relations avec l'environnement, ou encore la reconversion des écoles de villes en milieux permettant une éducation émancipatrice. Entre l'expérience de Vence et les synergies parisiennes, il y a objectivement une différence fondamentale, mais qui n'est pas nécessairement liée aux populations ou problématiques sociales en jeu. L'École Freinet a été créée en dehors de l'Instruction publique; les Freinet et leurs camarades ont été au bout de ce qu'ils voulaient faire, avec une liberté presque totale, dans un contexte particulier. Cette liberté en dehors de l'institution avait un coût, car il leur fallait subvenir à leurs besoins, 
ce qui rendait fragile cette aventure. Dans les années 1960, les militants parisiens officient dans l'Éducation Nationale dans des cursus qui dysfonctionnement complètement. Progressivement, la revue Éducateur Île-de-France devient le carrefour de mises en place d'alternatives dans les classes urbaines de la périphérie parisienne. Un problème récurrent semble être le carcan institutionnel figé de l'Éducation Nationale, notamment lorsqu'il s'agit de ces contextes spécifiques. Fonvieille parle de «structures paralysantes» de l'institution, en procédant à une mise en opposition du contexte des vieilles écoles rurales:

C'est dans la cadre intime du village que les expériences de Freinet et de ses compagnons ont pu se dérouler. Dans nos écoles-casernes, nous ne devons qu'à la ténacité de quelques-uns d'avoir pu introduire un peu de vie, par les techniques Freinet, dans des classes sclérosées au sein d'un corps figé (Fonvieille, 1961b, p. 6).

Bien qu'idéalisant le passé de l'École de Vence, Fonvieille et ses camarades œuvrent dans des conditions particulières, rendues également difficiles par l'administration et l'institution:

Nous luttons dans nos classes de villes, dans notre Groupe, dans cette revue, contre «l'encouragement permanent à l'ignorance et à un aveugle traditionalisme pédagogique», que constituent les gardes-fous hiérarchisés d'une administration fonctionnarisée à l'extrême (Fonvieille, 1961b, p. 6).

Le manque de temps, de matériel, le nombre élevé d'élèves font que l'application des techniques Freinet est jugée problématique (Reuge, 1963a, p. 35). Plusieurs comptes-rendus montrent concrètement comment changer l'organisation spatiale de sa classe, avec les moyens du bord, dans un établissement qui ne peut guère évoluer. D'autres textes proposent d'ailleurs de suivre une progression raisonnée et stratégique pour que les changements soient acceptés et réels. C'est le cas d'un texte de Lucien Reuge délimitant un "plan de modernisation»:

Le problème de l'inspection dans les classes pratiquant les techniques Freinet nous a conduits, au cours des travaux de la commission qui s'est réunie au Congrès de Niort, à établir un plan qui puisse permettre à l'inspecteur de situer à quelle étape est arrivé le maître dans la modernisation de son enseignement (1963b, p. 5).

Sans une vision réaliste des conditions d'émergence de l'École Freinet à Vence, mais avec la certitude que le monde a définitivement évolué vers une civilisation urbaine moderniste, certains militants comme Fonvieille ne réfléchissent qu’à partir d'un postulat de l'évidence de l'évolution:

Avec l'évolution de la société se produit inéluctablement une évolution de l'éducation puisque c'est au contact des conditions de vie et aussi en fonction des besoins que se déterminent les courants éducatifs (Fonvieille, 1960a, p. 1). À la recherche de nouveaux cadres de pensée, certains de ces militants fréquentent différents réseaux, s'ouvrent à de nouvelles figures intellectuelles à l'heure où 
les sciences humaines se développent et où les relations interindividuelles dans les groupes deviennent une préoccupation de premier plan, tant d'un point de vue pratique que théorique. Dès 1961, des instituteurs, dont plusieurs du Mouvement Freinet, s'organisent dans un collectif qui prend le nom de Groupe Techniques Éducatives (GTÉ). Dans ce contexte, Oury se distingue par une conception thérapeutique de l'éducation qui n'est pas étrangère à ses liens avec l'expérience de la clinique de La Borde, berceau de la psychothérapie institutionnelle, et dans laquelle œuvra son frère Jean Oury (1924-2014). Quelques temps plus tard, le colloque de Royaumont qui a lieu en deux temps lors de l'année 1962 à l'Abbaye de Royaumont a constitué aux yeux de beaucoup «un moment fondateur de l'analyse institutionnelle» (Schaepelynck, 2018, p. 60). En parallèle à ce colloque et ses enjeux, les figures influentes de Michel Lobrot (1924-2019), agrégé de philosophie devenu professeur au Centre de Pédagogie spéciale de Beaumont-sur-Oise, et Georges Lapassade (1924-2008), agrégé de philosophie et promoteur de l'analyse institutionnelle, contribuent dans le champ pédagogique à faire émerger la pédagogie institutionnelle autogestionnaires.

Pendant plusieurs années, la région francilienne est le lieu d'intenses réflexions pédagogiques et politiques, donnant lieu à des hybridations surprenantes. Le sociologue René Lourau (1933-2000) relate dans L’illusion pédagogique (1969) qu'en mars 1965 une réunion à la Mutualité avait été l'aboutissement de multiples contacts entre les militants de Socialisme ou Barbarie 6 et le "Groupe de Pédagogie institutionnelle», avec le sentiment qu'avant-garde pédagogique et avant-garde politique pouvaient s'entremêler. Lourau écrit:

Aussi étonnant que cela paraisse, le G.P.I. mêlant la critique du savoir magistral au projet d'autogestion et de révolution permanente, s'est manifesté un temps comme «rogero-trotskyste»! (Lourau, 1969, p. 49).

Dans ces réseaux, on retrouve également des membres de l'Association pour la Recherche et l'Intervention Psychosociologique, mais également des instituteurs du groupe Techniques Éducatives, dont Monique Labat, Raymond Fonvieille, Bernard Bessières, et des professeurs du Groupe de Pédagogie Institutionnelle, dont Lobrot, Lourau (p. 51).

\section{Réactions, conflits et paradoxes}

Au fur et à mesure des années 1950 puis 1960, le champ que constitue le Mouvement Freinet s'organise autour d'une tension entre ceux que l'on pourrait qualifier de «détenteurs d'un capital spécifique» et les autres, les «moindres possesseurs du capital spécifique» (Bourdieu, 2016, p. 1103). Le capital spécifique «qui paie» est le capital «Freinet» et qui renvoie à l'usage de techniques, à une référence précise à l'expérience de Vence, une conscience politique, un mode de vie, et une certaine manière de faire dialoguer des savoirs très différents (marxisme, Éducation nouvelle, philosophie indienne, médecine chinoise, biologie). Le plus 
proche de ce capital est Freinet lui-même, puisque le mouvement, les techniques et l'expérience originelle portent son nom. Co-auteurs de cette pédagogie spécifique, Élise et Célestin Freinet sont les mieux placés, en théorie, pour évaluer ce qui est, ou non, dans la ligne de ce qui a été fait jusqu'alors. Cependant, deux problèmes se posent: le premier, c'est que la stratégie de communication des Freinet apporte autant d'avantages que d'inconvénients; le second, c'est qu'entre détenteurs du capital «Freinet» et moindres possesseurs de ce capital se trouve une masse de plus en plus grande qui arbitre cette tension alors que le rapport de forces interne dans le mouvement est de plus en plus incertain.

\section{L'isolement des Freinet}

En 1956, Freinet entre dans sa septième décennie de vie. D'une certaine manière, les Freinet viennent d'une époque où le monde se divise en deux catégories: la bourgeoisie exploitante et le prolétariat exploité. Le poids du marxismeléninisme et du naturisme a souvent été sous-estimé lorsqu'il s'agissait de décrire l'émergence de la pédagogie des Freinet, mais il fut également négligé quand il fallut rendre compte de la position des Freinet dans différents réseaux plusieurs décennies plus tard. Les dramatiques événements du $20^{\mathrm{e}}$ siècle et les aléas de la vie humaine ont isolé le couple Freinet. Le constat dans les années 1950 est sans appel, nombre de proches des Freinet et de références emblématiques ${ }^{7}$ à l'œuvre dans leur trajectoire, ont littéralement disparu: Ovide Decroly (1874-1932), le médecin pédagogue de l'Éducation nouvelle; Vrocho (1892-1936), le gourou naturiste et guide révolutionnaire des Freinet; Meta Kraus-Fessel (1884-1940), proche des milieux libertaires allemands et militante de l'enfance prolétarienne; Erich Mühsam (1878-1934), poète libertaire emblématique des premières victimes du nazisme; Panaït Istrati (1884-1935), référence littéraire des réseaux révolutionnaires et symbole des calomnies staliniennes; mais également Paul Carton (1875-1947), figure majeure du naturisme peu connu pour son socialisme; ou encore Alexis Carrel (1873-1944), biologiste et écrivain marquant de l'Entre-deux-guerres qui se compromit pendant la Seconde guerre mondiale. Ce monde de pensée appartient dorénavant au passé.

Par ailleurs, cet isolement a été d'autant plus fort que le communisme des Freinet devient un communisme privé sans rattachement à un parti (Go \& Riondet, 2019). Les Freinet quittent le Parti Communiste Français en 1952 suite aux conflits les opposant aux tenants de ligne cultuelle du Parti, Georges Cogniot (1901-1978) et Roger Garaudy (1913-2012). À cette époque, les soutiens sont nombreux au sein du Mouvement, et en dehors, face à ces adversaires qui vivent pour certains leurs dernières années en position dominante. Au sein du Mouvement, les Freinet sont entourés de fidèles camarades, mais de plus en plus esseulés au fil du turnover générationnel qui se dessine dans les différents groupements pédagogiques, du premier ou du second degré (Riondet, 2013). L'heure est de moins en moins au marxisme tant celui-ci a été accaparé de manière dogmatique par les intellectuels du Parti ${ }^{8}$ dans une période 
de révisionnisme alors que simultanément l'Occident découvrait lentement les atrocités du stalinisme. Freinet n'est pas qu'isolé, il est souvent stigmatisé pour sa double étiquette d'instituteur communiste et rural (Riondet, 2015). Alors que la société française se caractérise de plus en plus par l'opposition entre d'une part, les poujadistes, issus des classes moyennes des entreprises indépendantes, venant de province et méfiants des élites parisiennes et, d'autre part, les mendésistes, partisans de la modernisation de la France, les positions des Freinet sont vite associées à un ruralisme nostalgique hostile à toute évolution et progrès technique (Go \& Riondet, 2019).

\section{Réactions et stratégies des Freinet}

Si la spécificité du contexte parisien est effectivement fondée, la situation est plus complexe qu'il n'y paraît. Fonvieille (1960b) évoque dans un de ses éditoriaux «les multiples visages de l'École Moderne» pour faire émerger l'évidence que deux classes Freinet puissent ne pas se ressembler. Évidemment, Freinet lui-même, en fidèle marxiste convaincu, avait défendu l'idée que les conditions matérielles peuvent influer sur la pédagogie et qu'on ne peut faire abstraction des conditions effectives d'exercice et d'accueil lorsque l'on réfléchit à la manière d'éduquer et de scolariser des populations. Néanmoins, si l'on qualifie une pédagogie d'un adjectif en particulier, ou qu'on l'assimile à un nom propre pour désigner une expérience, c'est que l'on peut distinguer ce qui est «Freinet» de ce qui n'est pas «Freinet», et que l'on peut trouver une proximité objective entre de nouvelles réalisations et l'expérience originelle. Dès 1958, cette ambiguïté entre, d'un côté, la légitimité à vouloir faire des choses en fonction d'un contexte particulier, et de l'autre côté, le maintien plus ou moins artificiel d'un nom propre («Freinet») est à l'œuvre dans le Mouvement Freinet.

Freinet a pleinement conscience des velléités de certains parisiens, mais il essaie dans un premier temps d'être stratège en valorisant dans la revue parisienne les différentes expérimentations qui peuvent avoir lieu sous la bannière «Freinet» tout en défendant simultanément une orthodoxie plus stricte dans d'autres revues (Riondet, 2015). Le potentiel d'audience et d'actions de l'IPÉM est important, Paris reste une place éminemment stratégique. Les demandes liées à l'IPÉM explosent en quelques mois. Le recensement du nombre de classes ayant sollicité une correspondance individuelle est éloquent puisque l'on passe de 34 demandes lors de l'année 1962-1963 à 99 pendant l'année 1963-1964. Dans le même laps de temps, le nombre d'adhérents à l'IPÉM croît de 112 à 196, tout comme le nombre d'abonnés à la revue «île-de-France»: de 64 à 144 (Faligand, 1964, p. 3). À la même époque, les négociations menées de longue date par Freinet pour qu'il y ait une école expérimentale Freinet en partenariat avec l'Institut Pédagogique National évoluent dans un premier temps, non en faveur d'un accord avec Vence, mais dans le cadre d'un partenariat avec une école de la région parisienne 9 . Pour des raisons stratégiques, Freinet ne peut se montrer que précautionneux, d'autant qu'au sein du Mouvement, l'hétérogénéité des 
tendances politiques prédomine: certains sont réformistes, parfois libertaires ou même anciens sympathisants communistes, sensibles aux questions écologiques, ou pas. Si l'essaimage «Freinet» est une réussite, notamment via des contextes professionnels parfois très différents (primaire, secondaire, écoles de perfectionnement, enseignement spécialisé, etc.), Freinet a une marge de manœuvre de plus en plus réduite ${ }^{10}$.

Tout en se réjouissant des actions parisiennes, Freinet tente de mettre en évidence la spécificité de l'expérience de Vence en essayant de fédérer autour de lui des universitaires et des personnalités pédagogiques dans l'aventure éditoriale Techniques de Vie ${ }^{11}$ (Riondet, 2015). Seulement, il faut reconnaître que l'objectivation scientifique de l'expérience de Vence et des pratiques pédagogiques et sociales qui la sous-tendent, qui était un des enjeux de la revue, se solde par un fiasco. La plupart des chercheurs et universitaires contactés sont en fait très éloignés, d'un point de vue épistémologique, de ce qui traversent l'expérience de Vence. Face à ce problème, il reste les publications mais la propagande pédagogique repose alors essentiellement sur les brochures et les ouvrages. Si ces documents se vendent convenablement, rien ne permet de dire qu'ils sont lus et compris de la manière dont les Freinet le souhaiteraient. En 1977, Élise s'indigne d'ailleurs, plusieurs années après la disparition de Freinet, du fait que les militants ne lisent plus, ce qui peut s'expliquer également par l'évolution des cadres sociétaux et culturels (Freinet, É., 1977). Nul doute que dans les années 1960, un film de propagande comme L'École Buissonnière produit encore davantage d'effets sur les individus que les brochures d'explicitation des Freinet. Freinet se retrouve donc dans une impasse, d'autant que même l'École Freinet à Vence est menacée par le révisionnisme pédagogique, lorsque Bonbonnelle, arrivé à Vence en septembre 1964, tente d'instaurer des pratiques issues des chantiers parisiens ${ }^{12}$ en opposition avec le système très précis à l'œuvre dans cette école.

Pour Freinet, le problème n'est pas que certains parisiens veulent faire autre chose, mais qu'ils le fassent sous la bannière "Freinet». Pour les parisiens, le problème général est la direction du Mouvement par Freinet. Ce dernier est accusé de "filtre culturel», empêchant les militants d'avoir accès à ce qui se passe à l'extérieur: psychologie, psychosociologie, rogérisme et autres recherches pédagogiques (Bonbonnelle et al., 1966). Le problème ne se pose pas qu'au niveau éditorial, puisque la structure de l'organisation est également critiquée. Les parisiens souhaiteraient une coopération sans direction, une autogestion totale. Seulement, comment imaginer un Mouvement Freinet dans lequel Freinet n'aurait pas un mot particulier à dire?

\section{Paradoxes}

Bénévent et Mouchet (2014) estiment que l'année 1958 aurait été une "année décisive» (p. 140) puisqu'Oury commence à avoir une idée de plus en plus précise de ce qui va devenir la pédagogie institutionnelle: s'entremêlent ici la question des effets thérapeutiques de l'école moderne, l'importance de l'incons- 
cient, la dynamique du transfert, le rôle de l'enseignant, la fonction des institutions etc. Ce réseau se serait autonomisé peu à peu de Freinet, pour deux raisons: d'une part, l'incompatibilité du modèle rural de l'École Freinet dans un environnement urbain; d'autre part, la réticence de Freinet à l'égard de la psychanalyse et des sciences humaines. S'il est exact qu'entre 1958 et 1961 une dissidence pédagogique et politique émerge peu à peu au sein du Mouvement Freinet, il est surprenant que ces suppositions discréditant la pensée des Freinet n'aient jamais été questionnées. Le continent de l'inconscient est au cœur de nombreuses réflexions de psychologues, de philosophes et de pédagogues dès le début du 20e siècle; Freinet conseillait à la fin des années 1930 à ses camarades la lecture de René Allendy (1989-1942), un médecin homéopathe et psychanalyste, Élise lisait René Laforgue (1894-1962) et échangeait avec certains de ses collaborateurs. La réticence des Freinet à l'égard des sciences humaines s'explique par la méfiance des modes théoriques susceptibles de détourner les réflexions et les luttes déjà engagées. Au sujet de la non-directivité, Freinet avait en son temps modéré certains enthousiasmes (Freinet, 1964). Seulement, à l'heure où explosent les sciences humaines et sociales, Freinet est à contre-courant, préférant des œuvres plus atypiques, comme celle de Teilhard de Chardin (1881-1955), rencontre complexe entre «l'eschatologie catholique» et le "progrès technique» (Borne, 1988, p. 43).

En réalité, la pensée des Freinet n'était pas si inadaptée aux questions en jeu. La problématisation naturiste qui caractérise l'accélération de la pensée des Freinet à partir de 1932 et qui fonde l'expérience de Vence (Riondet, 2016) s'adressait justement aux citadins exposés aux conditions de vie aliénantes de la civilisation industrielle. Le naturisme prolétarien n'est pas une idéologie de passage dans la trajectoire des Freinet car le «milieu paysager» est fondamentalement au cœur de la pédagogie de l'École Freinet à Vence (Go, 2007). En réalité, ce naturisme prolétarien est d'autant plus subversif que les années 1960 marquent l'émergence de la société de consommation autour des modes de vie que critique justement la pensée freinetienne des commencements. Oury, lui-même, en était conscient ${ }^{13}$. Lorsque les germes de la pédagogie institutionnelle prennent forme, parfois au sein même du Mouvement Freinet, avant de se diffuser dans d'autres directions, chaque protagoniste cherche à tirer profit de l'autre. Les futurs institutionnalistes s'appuient sur l'appareil que constitue originellement l'ICÉM, tout comme Freinet essaie de profiter de l'essor et l'enthousiasme qui entourent le Groupe Parisien, mais cette situation n'est tenable pour personne car il s'agit, en dernière instance, de pensées et de pratiques fondamentalement différentes. Ultime paradoxe: Freinet disparaît en 1966 alors que ses contradicteurs majeurs ont monté leurs propres structures, laissant un Mouvement Freinet orphelin d'un de ses fondateurs mais de plus en plus influencé par ces figures parisiennes. 


\section{Conclusion}

Le monde parisien du Mouvement Freinet est marqué par l'émergence de la pédagogie institutionnelle mais la rencontre manquée entre les jeunes militants parisiens et les Freinet peut s'expliquer par des raisons dépassant les querelles entre personnes. Il n'est pas illégitime de penser que la compréhension et le prolongement de certaines expériences de l'Éducation nouvelle influencées par des courants comme la théosophie ou le naturisme aient été rendues difficiles par l'évolution sociétale et certaines de ses caractéristiques (prédominance de l'urbanisme, progrès technologique et enjeux de croissance économique).

Dans les années 1960, la subtilité et la force de la pensée des Freinet deviennent l'affaire de cercles d'initiés, pendant que la pédagogie institutionnelle occupe le devant de la scène pédagogique en s'associant à la recherche pédagogique émergeante et aux sciences de l'éducation, sans que l'on s'interroge nécessairement sur les spécificités de chaque style de pensée. La pédagogie institutionnelle d'Oury et de Fonvieille procède d'une certaine interprétation de la «Pédagogie Freinet» bien loin de ce que nous révèle l'objectivation de la singularité de Vence dont l'ambition n'a jamais été de s'enfermer dans la catégorie rurale en dehors de l'Histoire. Il est arrivé qu'on souligne l'incapacité de Freinet à trouver et former des successeurs ${ }^{14}$. S'est-on demandé si certains individus avaient vraiment cherché à être réellement des disciples du maître?

La fidélité au jeune Freinet aurait induit un diagnostic différent de l'IPÉM sur la situation des parisiens: le problème n'est pas l'inadaptation des techniques Freinet au contexte francilien, mais le fait que celles et ceux que l'on pourrait qualifier de «malades scolaires» (Go et Riondet, 2019) sont victimes, en dernière instance, de leur mode de vie et de leurs conditions d'existence, ce qui exigerait un milieu éducatif radicalement différent du cadre traditionnel.

\section{Notes}

1 Pithiatiques, comitiaux, schizoïdes, mélancoliques, grands caractériels.

2 CAEA : Certificat d'Aptitude à l'Enseignement des enfants Arriérés

3 «Il n'est certes pas question dans des locaux exigus, accessibles par des escaliers insuffisants, avec des effectifs presque supérieurs à 35, de travailler dans les paisibles classes de campagne ou à l'École Freinet de Vence» (Éducateur Île-de-France, 1960, p. 12).

4 Par «franciliano-centrisme», nous désignons une tendance encore très actuelle en France de penser la situation en Île-de-France comme représentative du reste du territoire français. On parle généralement du "parisiano-centrisme» mais, dans le cas évoqué dans cet article, il s'agit plus souvent de communes situées en Île-de-France que dans Paris intra-muros.

5 Ce qui contribuera rapidement à accentuer les différends entre Oury et Fonvieille qui venaient de quitter le Mouvement Freinet.

6 «Socialisme ou Barbarie» est une organisation révolutionnaire créée en France par Cornelius Castoriadis (1922-1997) et Claude Lefort (1924-2010).

7 Et parfois peu compatibles entre elles.

8 C'est face à ce dogmatisme intellectuel, incarné notamment par Garaudy, qu'il faut lire l'émergence d'Althusser à la fin des années 1960 et sa volonté de relire Marx. 
9 Freinet arrivera finalement à ses fins lorsqu'une convention entre l'École Freinet de Vence, l'ICÉM et l'IPN fut officialisée quelques mois plus tard, ce qui était, de longue date, un des ses objectifs principaux.

10 Jasques Bens et Madeleine Freinet essaieront bien plus tard de faire déposer la marque «Freinet» à l'Institut National de la Propriété Industrielle pour se prémunir des récupérations et des déviations (Go \& Riondet, 2019).

11 Revue créée par Freinet en 1959, dont la parution s'arrête en 1964 (Riondet, 2015).

12 Par exemple la pratique de la récréation, incompatible avec la pensée des Freinet.

13 Oury écrit notamment: «Freinet et l'École Moderne ont proposé des techniques directement utilisables en milieu rural qui ont en ville un inconvénient majeur. Elles mènent directement à une reconsidération totale de la structure des écoles» (Oury, 1962, p. 3).

14 Ce qui est faux, puisque Freinet travaillait de façon très proche avec quelques instituteurs qu'il estimait tout particulièrement (Rauscher, Delbasty, Le Bohec...).

\section{Bibliographie}

Action psychologique de la Coopérative dans la Classe Moderne. (1958). Troisième réunion psycho-pédagogique organisée le 25 septembre 1958 par l'IPEM. Fonds Go.

Asselain, J.-C. (1984). Histoire économique de la France du XVIIIe siècle à nos jours. Paris, France: Seuil.

Bénévent, R., \& Mouchet C. (2014). L'école, le désir et la loi: Fernand Oury et la pédagogie institutionnelle. Nîmes, France: Champ Social.

Bonbonnelle, A., Faligand, M., \& Gilbert, H. (1966). Contribution à l'histoire du mouvement Freinet. Paris, France: Imprimerie EAM.

Borne, D. (1988). Histoire de la Société Française depuis 1945. Paris, France: Armand Colin.

Bourdieu, P. (2015). Sociologie générale. Volume 1. Paris, France: Seuil/Raisons d'agir.

Bourdieu, P. (2016). Sociologie générale. Volume 2. Paris, France: Seuil/Raisons d'agir.

Bruliard, L., \& Schlemminger, G. (2012). Le Mouvement Freinet: des origines aux années quatre-vingt. Paris, France: L'Harmattan.

Dubois, A. (2019). Histoires de la pédagogie institutionnelle. Les monographies. Nîmes, France: Champ Social.

Dupeux, G. (1972). La société française 1789-1879. Paris, France: Armand Colin.

Éducateur Île-de-France. (1960). Vers des solutions mixtes en groupe de ville. Éducateur Île-de-France, 36, 12-13.

Faligand, M. (1961). Écoles de villes. Éducateur Île-de-France, 42, 25-26.

Faligand, M. (1963). Classes de perfectionnement. Educateur Île-de-France, 52, 17-18.

Faligand, M. (1964). Vie de l'IPEM. Éducateur Île-de-France, 57-58, 3-4.

Faligand, M. (1965). Expériences d'autogestion à l'école primaire. Cahiers Pédagogiques, 55, 49-52.

Fonvieille, R. (1958/1959). Nous sommes - mais nous ne sommes pas seuls. Éducateur Île-deFrance, 18/19, 1-2.

Fonvieille, R. (1960a). Un courant irréversible. Educateur Île-de-France, 30, 1-2.

Fonvieille, R. (1960b). Les multiples visages de l'École Moderne. Éducateur Île-de-France, $32 / 33,1-2$.

Fonvieille, R. (1961a). À propos de schématisations abusives. Éducateur Île-de-France, 36, 7-9.

Fonvieille, R. (1961b). Des structures paralysantes. Éducateur Île-de-France, 39, 5-7.

Fonvieille, R. (1961c). Pour la petite histoire. Éducateur Île-de-France, 39, 8-11.

Fonvieille, R. (1989). L'aventure du Mouvement Freinet. Paris: Klincksieck.

Freinet, C. (1946a). Questions urgentes. L'Éducateur, 9, 137-140.

Freinet, C. (1946b). Prises de position. L'Éducateur, 10, 169-180.

Freinet, C. (1946c). Le Conseil d'administration de la CEL a pris d'importantes décisions. L'Éducateur, 11, Couverture 2. 
Freinet, C. (1964). L’attitude non-directive en pédagogie. Techniques de Vie, 30/31, 28-30.

Freinet, É. (1977). Le message d'Élise Freinet. Bulletin de la Société Binet-Simon, 577, 185-189. Go, H. L. (2007). Freinet à Vence. Rennes, France: PUR.

Go, H. L. (2015). Les deux créations de l'Institut Freinet de Vence. In A. D. Robert \& B. Garnier (Éd.), La pensée critique des enseignants. Éléments d'histoire et de théorisation (pp.77-90). Rouen, France : PURH.

Go, H. L., \& Riondet, X. (2019). A côté de Freinet. Nancy, France : PUN-EDULOR.

Hess, R. (1975). La pédagogie institutionnelle aujourd'hui. Paris, France: Delage.

Hess, R., \& Savoye, A. (1993). L'analyse institutionnelle. Paris, France: PUF.

Lourau, R. (1969). Lillusion pédagogique. Paris, France: Epi.

Michaud, G. (1969). Analyse institutionnelle et pédagogie [Numéro spécial]. Recherches, 9.

Oury, F. (1959). Conserver n'est pas défendre. Éducateur Île-de-France, 21, 1-2.

Oury, F. (1962). L'adaptation de l'École Publique est la seule solution. Éducation et techniques, 4, $1-4$.

Oury, F., \& Pain, J. (1972). Chronique de l'École-Caserne. Paris, France: Maspero.

Pain, J. (1979). Une formation à la pratique de l'institutionnel. Pédagogie institutionnelle, pratiques institutionnelles et formation. Thèse en sciences de l'éducation, Université Paris X Nanterre, France.

Reuge, L. (1963a). Écoles de villes. Éducateur Île-de-France, 50/51, 35-42.

Reuge, L. (1963b). Propositions. Éducateur Ille-de-France, 52, 5-6.

Riondet, X. (2013). (2013). Les origines des Cahiers pédagogiques en 1945. Les Sciences de l'éducation. Pour l'Ère nouvelle, vol. 46, 3, 113-135.

Riondet, X. (2015). La revue Techniques de Vie (1959-1964) ou l'impossibilité des collaborations. Un épisode entre histoire du militantisme et histoire des sciences de l'éducation. Penser l'éducation, 36, 119-143.

Riondet, X. (2016). Une expérience d'éducation régénératrice: l'École Freinet à Vence (19341939). Les Études Sociales, 163, 151-171.

Riondet, X. (2019). L'expérience Vrocho à Nice. Controverses et résistances du quotidien au coeur de l'évolution des normes. Rouen, France: PURH.

Savoye, A. (2004). L'Éducation nouvelle en France. De son irrésistible ascension à son impossible pérennisation (1944-1970). In A. Ohayon, D. Ottavi, \& A. Savoye (Éds.), L'Éducation nouvelle, histoire, présence et devenir (pp. 235-269). Bern, Suisse: Peter Lang.

Schaepelynck, V. (2018). L'institution renversée. Folie, analyse institutionnelle et champ social. Paris, France: Eterotopia.

Mots-clés: Mouvement Freinet, pédagogie institutionnelle, réception, controverses, Vence 


\section{Rezeption und Verwässerung des Beitrags von Freinet's Denken. Der Fall pädagogischer Aktivisten in Paris nach 1945}

\section{Zusammenfassung}

Die Reformpädagogik umfasst unterschiedliche Strömungen und Ansätze. Wie haben sich diese Strömungen im Verlauf der Jahre weiter entwickelt? Im vorliegenden Beitrag untersuchen wir anhand des Beispiels der französischen FreinetBewegung, wie die ursprünglich von Freinet entwickelten Ideen aus den 1930er Jahren von militanten Pariser Gruppen der 1960er Jahre rezipiert wurden. Das Ergebnis ist eine sorgfältige historisch begründete Analyse der internen Kontroversen und Divergenzen der Freinet-Bewegung - in einer Zeit, in der sich eine neue institutionelle Pädagogik zu etablieren beginnt.

Schlagworte: Freinet-Bewegung, institutionelle Pädagogik, Rezeption, Kontroverse, Vence

\section{Ricezione e diluizione degli apporti dei Freinet. Il caso dei militanti pedagogici parigini del secondo dopoguerra}

Riassunto

L'Educazione Nuova è caratterizzata da diverse correnti e scuole di pensiero. In che modo questi movimenti si sono evoluti e sono perdurati nel corso degli anni? In questo contributo si prende ad esempio il movimento francese Freinet. Attraverso l'analisi del processo di ricezione del pensiero originale dei sostenitori di Freinet - degli anni '30 - da parte dei militanti parigini degli anni ' 60 , è proposta un'interpretazione delle controversie e delle divergenze interne al Movimento Freinet agli albori dell'emergere di una nuova avventura della pedagogia istituzionale.

Parole chiave: Movimento Freinet, pedagogia istituzionale, accoglienza, polemica, Vence 


\section{Reception and dilution of the Freinets' philosophical contribution.Case study: Parisian pedagogical activists after 1945}

\section{Summary}

Progressive Education stems from different schools and trends of thought. How have these collectives evolved and lived on over the years? This article focuses on the example of the French Freinet Movement. Examining how the original Freinet principles of the 1930s were received by the Parisian militants of the 1960 s, it offers a reasoned analysis of controversies and divergences within the Freinet Movement at the dawn of a new emerging adventure in institutional pedagogy.

Keywords: Freinet Movement, institutional pedagogy, reception, controversies, Vence

Xavier Riondet. Xavier Riondet. Après des études en sciences de l'éducation à l'Université Nancy2, et des recherches en histoire de l'éducation à l'Université de Genève, il est actuellement maittre de conférences en sciences de l'éducation à l'Université de Lorraine. Ses travaux portent sur l'évolution des valeurs éducatives durant le 20e siècle et en particulier sur l'analyse de la contribution des normativités éducatives alternatives à ces processus.

Université de Lorraine, CLSH, Sciences de l'éducation, 23, boulevard Albert 1er BP 60446, 540001 Nancy Cedex France.

E-Mail: xavier.riondet@univ-lorraine.fr 